\title{
A novel scale for describing visual outcomes in patients following resection of lesions affecting the optic apparatus: the Unified Visual Function Scale
}

\author{
Serge Makarenko, MD, Vincent Ye, Peter A. Gooderham, MD, FRCSC, and \\ Ryojo Akagami, MD, MHSc, FRCSC
}

Division of Neurosurgery, Vancouver General Hospital, University of British Columbia, Vancouver, British Columbia, Canada

\begin{abstract}
OBJECTIVE Historically, descriptions of visual acuity and visual field change following intracranial procedures have been very rudimentary. Clinicians and researchers have often used basic descriptions, such as "improved," "worsened," and "unchanged," to describe outcomes following resections of tumors affecting the optic apparatus. These descriptors are vague, difficult to quantify, and challenging to apply in a clinical perspective. Several groups have attempted to combine visual acuity and visual fields into a single assessment score, but these are not user-friendly. The authors present a novel way to describe a patient's visual function as a combination of visual acuity and visual field assessment that is simple to use and can be used by surgeons and researchers to gauge visual outcomes following tumor resection.
\end{abstract}

METHODS Visual acuity and visual fields were combined into 3 categories designed around the definitions of legal blindness and fitness to drive in Canada. The authors then applied the scale (the Unified Visual Function Scale, or UVFS) to their previously published case series of perisellar meningiomas to document and test overall visual outcomes for patients undergoing tumor resection. The results were compared with previously documented visual loss scales in the literature.

RESULTS Using the UVFS the authors were able to capture the overall visual change; the scale was sensitive enough to define the overall visual improvement or worsening quantitatively, using categories that are clinically relevant and understandable.

CONCLUSIONS The UVFS is a robust way to assess a patient's vision, combining visual fields and acuity. The implementation of pre- and postoperative assessment is sensitive enough to assess overall change while also providing clinically relevant information for surgeons, and allows for comparisons between treatment groups.

https://thejns.org/doi/abs/10.3171/2017.6.JNS17707

KEY WORDS visual field; visual acuity; scale; combined; outcome; oncology

$\mathrm{O}$ RBITAL tumors, as well as intracranial lesions affecting the optic apparatus, are commonly approached by a multidisciplinary team of skull base surgeons, ophthalmologists, and otolaryngologists. Representing $5 \%-15 \%$ of all intracranial lesions, they most commonly include pituitary tumors, meningiomas, and fibrous dysplasia, as well as vascular, inflammatory, and metastatic lesions. ${ }^{13,21,35}$ The difficulty with resection of these lesions has been mainly attributed to the high risk of visual pathway and pituitary apparatus involvement, in addition to vascular encasement and cavernous sinus invasion. Surgical approaches are mainstays of definitive treatment, targeting gross-total resection while preserving visual function. Subfrontal, pterional, and supraorbital approaches (with and without various osteomomies), as well as endonasal endoscopic resection, remain standard for resection of these lesions. . $^{32,37}$

While describing visual outcomes in the resection of these lesions, many authors have selected a purely qualitative approach.,14,24 Descriptors such as "improved," "worsened," and "unchanged" are vague and difficult to apply in a clinical setting. ${ }^{36}$ More importantly, these terms pose a significant challenge when attempting to quantify outcomes following resection of these lesions. There is a need to consider several variables involved, such as visual fields and acuity, monocular or binocular involvement, and varying visual changes in individual eyes. ${ }^{33}$ Early studies examined visual acuity only. ${ }^{11,18}$ More recent case series have investigated visual acuity and visual fields as discrete variables. ${ }^{8,9,17,22,23}$ Rosenstein and Symon attempted to com-

ABBREVIATIONS ADVS = Activities of Daily Vision Scale; UVFS = Unified Visual Function Scale; VFQ-25 = 25-Item Visual Function Questionnaire; VIQ = visual improvement quotient; VIS = visual impairment score; VL = visual loss.

SUBMITTED March 20, 2017. ACCEPTED June 5, 2017.

INCLUDE WHEN CITING Published online January 12, 2018; DOI: 10.3171/2017.6.JNS17707. 
TABLE 1. UVFS grades based on definition of legal blindness and requirements for fitness to drive

\begin{tabular}{|c|c|c|c|}
\hline \multirow[b]{2}{*}{ Visual Field } & \multicolumn{3}{|c|}{ Visual Acuity } \\
\hline & $20 / 20-20 / 50$ & $<20 / 50-20 / 200$ & $<20 / 200$ \\
\hline$\geq 120^{\circ}$ along horizontal axis $\& \geq 15^{\circ}$ above \& below the level of fixation & Grade A & Grade B & Grade C \\
\hline$<120^{\circ}$ along horizontal axis or $<15^{\circ}$ above \& below level of fixation, but $\geq 20^{\circ}$ & Grade B & Grade B & Grade C \\
\hline$<20^{\circ}$ & Grade C & Grade C & Grade C \\
\hline
\end{tabular}

UVFS grades: $\mathrm{A}=$ good vision; $\mathrm{B}=$ adequate vision $\mathrm{C}=$ poor vision .

bine visual acuity and visual fields to attribute an overall visual loss (VL) score while describing visual outcomes. ${ }^{33}$ The German Ophthalmological Society describes a visual impairment score (VIS) as a calculation combining visual fields and visual acuity. ${ }^{20}$ Fahlbusch and Schott used this scale to quantify visual outcomes in their resection of tuberculum sellae meningiomas. ${ }^{14}$ Unfortunately, these scoring systems are cumbersome and impractical for routine use by surgeons and clinical researchers. Other scales, such as the 25-Item Visual Function Questionnaire (VFQ-25) or Activities of Daily Vision Scale (ADVS), have been used in attempts to correlate visual function with quality of life, but these are also unsuitable for routine clinical use. . $6,27^{27}$

We present a novel Unified Visual Function Scale (UVFS) combining visual acuity and visual fields into a single assessment, while incorporating the definitions of legal blindness and "fitness to drive" to quantify visual outcomes. We attempted to design an assessment scale that is detailed enough to capture changes in visual acuity and fields, while providing a global assessment of patient visual function that both is clinically relevant and takes into account features important for patient quality of life. This scale can be used by surgeons and researchers to gauge visual outcomes following resection of tumors affecting the optic apparatus. We applied our scale to the previous case series of 53 perisellar meningiomas removed at our institution between 2001 and 2013 by the senior author (R.A.).

\section{Methods}

\section{Development of the UVFS}

The UVFS was designed as an attempt to reflect clinically relevant factors that directly impact patient quality of life (Table 1). Three grades (A-C) were assigned based on the definitions of legal blindness and fitness to drive. The normal binocular visual field is $135^{\circ}$ vertically and $180^{\circ}$ horizontally from the fixed point. ${ }^{6}$ The Canadian Association of Optometrists defines legal blindness as visual acuity worse than or equal to 20/200 with best correction in the better eye, or a visual field extent of less than $20^{\circ}$ in diameter horizontally. ${ }^{5}$ For driving a private vehicle, the Canadian Medical Association recommends a visual acuity greater than 20/50 and a visual field at least 120 continuous degrees along the horizontal meridian and 15 continuous degrees above and below fixation, examined with both eyes open together.6,7,28 Grade A was deemed good vision, meeting the requirements to drive. Grade B did not meet the threshold to drive a private vehicle and was designated as "adequate vision," but was better than "poor vision" (grade C), defined as visual acuity and visual fields meeting the definition of legal blindness (Table 1).

\section{Patient Population}

We then reviewed our case series of 53 patients who were diagnosed with perisellar meningiomas and underwent resection at our center between January 2001 and December 2013. ${ }^{25}$ Microsurgical resection of the lesions was performed via a unilateral frontotemporal craniotomy with orbital osteotomy, depending on the side with worse vision, and an extradural decompression of the optic canal with a resection of the clinoid process prior to removal of the tumor. Analysis of clinical records and radiographic findings was previously performed in detail. All of the patients were evaluated by a neuroopthalmologist before and after surgery, with a thorough assessment of visual acuity and visual fields. These assessments were documented and compared against those recorded in clinic notes by the senior surgeon to ensure consistency.

\section{Testing the Scale}

Patient visual acuity was tested and recorded in each eye individually with the best-correcting glasses for both eyes. Funduscopy was also performed where applicable and results recorded. Humphrey perimetry visual fields up to $30^{\circ}$ temporally and nasally (24-2 or 30-2 where appropriate) were performed in all patients using the automated Humphrey Zeiss field analyzer (Carl Zeiss Meditec). Those patients who had visual field deficits had Goldmann visual fields recorded. The results of visual acuity and visual field tests were then combined for each eye individually, and each eye assigned a UVFS grade of A, B, or C.

Our case series of perisellar meningiomas compared lesions based on "simple" or "complex" anatomy depending on whether the lesions were appropriate for endoscopic or open microsurgical resection, respectively. The rationale for the breakdown of the patients into each group has been previously described. ${ }^{25}$ Characteristics that influenced selection criteria included tumor size, vascular encasement, optic canal involvement, and tumor shape. The lesions that included significant lateral extension or those tumors that had extensive vascular encasement were deemed more suitable for open resection and were designated as "complex anatomy." Those lesions that were believed to be easily accessible through an endoscopic approach were designated as "simple anatomy." To assess the sensitivity of the UVFS to quantitatively grade changes in visual quality before and after surgery, as well as within case cohorts, we selected 10 patients with the greatest variation in pre- and postoperative vision from each simple and complex anatomy group for illustrative purposes. For qualitative comparison and contrast, these 20 patients were also graded with VISs and the visual im- 
TABLE 2. Points and resulting percentages of VL for use in the VL point scoring system*

\begin{tabular}{|c|c|c|c|}
\hline \multirow[b]{2}{*}{ Extent of VL } & \multirow[b]{2}{*}{ Score } & \multicolumn{2}{|c|}{ VL $(\%)$} \\
\hline & & Binocular & Monocular \\
\hline \multicolumn{4}{|l|}{ Visual field } \\
\hline $\begin{array}{l}\text { Quandrantanopsia or less, small } \\
\text { scotoma }\end{array}$ & 1 & 5 & 10 \\
\hline Hemianopsia, large scotoma & 2 & 10 & 20 \\
\hline$\geq 75 \%$ visual field loss & 3 & 15 & 30 \\
\hline \multicolumn{4}{|l|}{ Visual acuity } \\
\hline Loss of $6 / 9, \mathrm{~J} 6, \mathrm{~N} 8$ & 1 & 5 & 10 \\
\hline Loss of 6/12, J8, J10, N10, N12 & 2 & 10 & 20 \\
\hline $\begin{array}{l}\text { Loss of 6/18, 6/24, J12, J14, J16, } \\
\text { N14, N18 }\end{array}$ & 2.5 & 12.5 & 25 \\
\hline $\begin{array}{l}\text { Loss of 6/36, 6/60, J18, J20, } \\
\quad \text { N24, N36, N48 }\end{array}$ & 3 & 15 & 30 \\
\hline Loss of $1 / 60, N 48$ & 5 & 25 & 50 \\
\hline Finger counting & 6 & 30 & 60 \\
\hline Hand movements & 7 & 35 & 70 \\
\hline Light perception & 9 & 45 & 90 \\
\hline Blindness & 10 & 50 & 100 \\
\hline $\begin{array}{l}\text { Isolated monocular blindness w/ } \\
\text { no VL in opposite eye }\end{array}$ & 10.5 & 52.5 & - \\
\hline
\end{tabular}

* The VIQ calculation was derived from VL pre- and postoperatively. Adapted and reprinted with permission from Rosenstein and Symon. ${ }^{33}$ provement quotient (VIQ) previously documented in the literature (Table 2, Fig. 1). To calculate the VIQ, points are totaled for each eye and the percentage of VL is calculated. Isolated monocular blindness with no loss of vision in the opposite eye was given a score of $52.5 \%$ to take into the account the loss of stereoscopic vision (Table 2): ${ }^{14,33}$ $\mathrm{VIQ}=\left(\mathrm{VL}_{\text {preop }}[\%]-\mathrm{VL}_{\text {postop }}[\%]\right) / \mathrm{VL}_{\text {preop }}(\%)$.

\section{Statistical Analysis}

Patient visual acuity and visual fields were analyzed using descriptive statistics. A p value $<0.05$ was considered statistically significant. We tabulated 1-eye data for illustrative purposes, but given that we cannot account for intereye correlation in this retrospective design, statistical analysis was not performed..$^{31}$ The chi-square test was used for the categorical variables, but when they did not meet the assumptions of minimum expected frequencies, Fisher's exact tests were used. Continuous data were tested using the Wilcoxon rank-sum test as they were statistically significant, nonnormally distributed data as tested using the Benjamini-Hochberg procedure to ensure a false discovery rate no higher than 5\%.,16 Data were collected using Microsoft Excel (version 2010, Microsoft Corp.). The statistical analyses were conducted using JMP (version 9.0.1, SAS Institute Inc.).

\section{Results}

\section{Patient Population}

We retrospectively reviewed the medical files and ophthalmology reports of patients with perisellar meningiomas treated with a frontotemporal craniotomy at our

\section{Visual acuity}

\begin{tabular}{|l|l|l|l|l|l|l|l|l|l|l|l|l|l|l|l|}
\hline & & 1,0 & 0,8 & 0,63 & 0,5 & 0,4 & 0,32 & 0,25 & 0,2 & 0,16 & 0,1 & 0,08 & 0,05 & 0,02 & 0 \\
\hline & $5 / 5$ & $5 / 6$ & $5 / 8$ & $5 / 10$ & $5 / 12$ & $5 / 15$ & $5 / 20$ & $5 / 25$ & $5 / 30$ & $5 / 50$ & $1 / 12$ & $1 / 20$ & $1 / 50$ & 0 \\
\hline 1,00 & $5 / 5$ & 0 & 2 & 4 & 6 & 8 & 10 & 12 & 15 & 17 & 20 & 22 & 25 & 27 & 30 \\
\hline 0,8 & $5 / 6$ & 2 & 4 & 8 & 10 & 12 & 15 & 17 & 20 & 22 & 25 & 27 & 30 & 32 & 35 \\
\hline 0,63 & $5 / 8$ & 4 & 8 & 15 & 17 & 20 & 22 & 25 & 27 & 30 & 32 & 35 & 37 & 40 & 42 \\
\hline 0,5 & $5 / 10$ & 6 & 10 & 17 & 20 & 22 & 25 & 27 & 30 & 32 & 35 & 40 & 42 & 45 & 47 \\
\hline 0,4 & $5 / 12$ & 8 & 12 & 20 & 22 & 25 & 30 & 32 & 35 & 37 & 40 & 42 & 47 & 50 & 52 \\
\hline 0,32 & $5 / 15$ & 10 & 15 & 22 & 25 & 30 & 35 & 40 & 45 & 47 & 50 & 55 & 57 & 60 & 62 \\
\hline 0,25 & $5 / 20$ & 12 & 17 & 25 & 27 & 32 & 40 & 50 & 52 & 55 & 57 & 60 & 65 & 67 & 70 \\
\hline 0,2 & $5 / 25$ & 15 & 20 & 27 & 30 & 35 & 45 & 52 & 55 & 57 & 60 & 65 & 70 & 75 & 80 \\
\hline 0,16 & $5 / 30$ & 17 & 22 & 30 & 32 & 37 & 47 & 55 & 57 & 60 & 65 & 70 & 75 & 80 & 85 \\
\hline 0,1 & $5 / 50$ & 20 & 25 & 32 & 35 & 40 & 50 & 57 & 60 & 65 & 75 & 80 & 85 & 87 & 90 \\
\hline 0,08 & $1 / 12$ & 22 & 27 & 35 & 40 & 42 & 55 & 60 & 65 & 70 & 80 & 85 & 90 & 92 & 95 \\
\hline 0,05 & $1 / 20$ & 25 & 30 & 37 & 42 & 47 & 57 & 65 & 70 & 75 & 85 & 90 & 98 & 100 & 100 \\
\hline 0,02 & $1 / 50$ & 28 & 32 & 40 & 45 & 50 & 60 & 67 & 75 & 80 & 87 & 92 & 100 & 100 & 100 \\
\hline 0 & 0 & 30 & 35 & 42 & 47 & 52 & 62 & 70 & 80 & 85 & 90 & 95 & 100 & 100 & 100 \\
\hline
\end{tabular}

Visual field defect

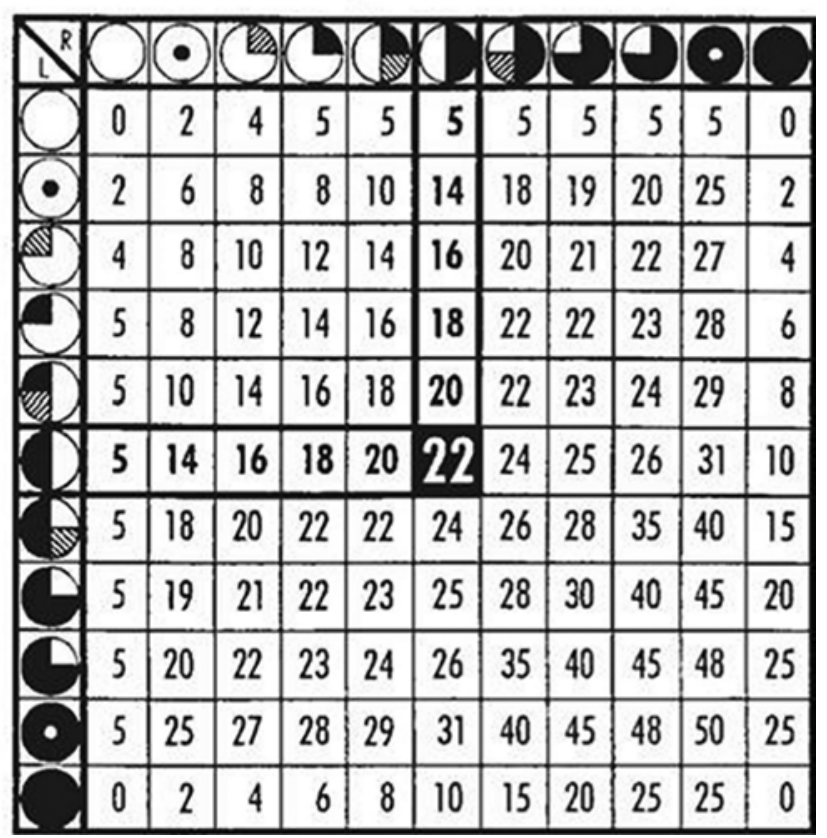

FIG. 1. Tables of visual acuity and visual field defect used for calculation of the VIS as defined by the German Ophthalmological Society. ${ }^{20}$ The marked values illustrate calculations made for a patient with a visual acuity of $0.4(4 / 10)$ in the left eye and $0.2(2 / 10)$ in the right eye, along with a bitemporal visual field defect. When added, the values 35 and 22 total 57 , representing the VIS. The range is $0-100$, with 100 representing greatest visual impairment. From the German Ophthalmological Society. 
TABLE 3. Detailed pre- and postoperative UVFS grades of patients with perisellar meningiomas

\begin{tabular}{ccc}
\hline UVFS Grade & Preop & Postop \\
\hline Simple anatomy & & \\
\hline A & $25(78.1)$ & $28(87.5)$ \\
\hline B & $2(6.3)$ & $4(12.5)$ \\
\hline C & $5(15.6)$ & $0(0.0)$ \\
\hline Complex anatomy & & \\
\hline A & $35(56.5)$ & $37(59.7)$ \\
\hline B & $11(17.7)$ & $12(19.4)$ \\
\hline C & $16(25.8)$ & $13(20.9)$ \\
\hline
\end{tabular}

Data includes 94 eyes in 47 patients: 32 with simple anatomy and 62 with complex anatomy.

* Values are presented as the number of patients (\%).

center between January 2001 and December 2013 by the senior author. These 53 patients were previously reviewed with respect to tumor anatomy and suitability for endoscopic resection. ${ }^{25}$ Detailed visual acuity and visual field data were documented by an ophthalmologist as well as the surgeon in $47(88.7 \%)$ of the patients. We previously documented a greater number of patients with simple anatomy who had improvements in visual acuity $(7 / 10,70.0 \%)$ and visual fields $(7 / 9,77.7 \%)$, while fewer patients with complex anatomy experienced better visual acuity or visual field function (7/26 [26.9\%] and 8/25 [32.0\%], respectively) after surgery. The conclusion was that those patients with simple anatomy had better qualitative overall visual outcomes after resection.

\section{UVFS}

Combining both the visual acuity and visual field tests using the UVFS, we were again able to quantitatively demonstrate that preoperatively, patients with perisellar meningiomas with simple anatomy had better visual function with more A grades (78.1\% vs 56.5\%; Table 3). We also confirmed our previous findings in those patients with tumors with complex anatomy who had objectively poor visual function (C grade) prior to resection (15.6\% vs $25.8 \%$ ). Additionally, we quantified postoperative visual function using the UVFS, which demonstrated an overall shift toward visual improvement in both surgical cohorts, with a difference in the higher number of patients with poor vision (C grade) with complex lesions $(0.0 \%$ vs $20.9 \%$; Table 3 , Fig. 2). Graphic representation of the net overall changes in visual function again qualitatively demonstrates the trend toward visual improvement in the postoperative period following resection of perisellar meningiomas (Fig. 2). There was no significant difference in postoperative follow-up at the time of the ophthalmological assessment (36.3 vs 21.7 months, $\mathrm{p}=0.05$ ).

\section{Comparison of the UVFS to the VIQ and German Ophthalmological Society Score (VIS)}

Clinicians have several choices when assessing visual fields and visual acuity, assessing them either individually or in combination. A sample of 10 simple and 10 complex meningioma cases was scored using the UVFS and the 2
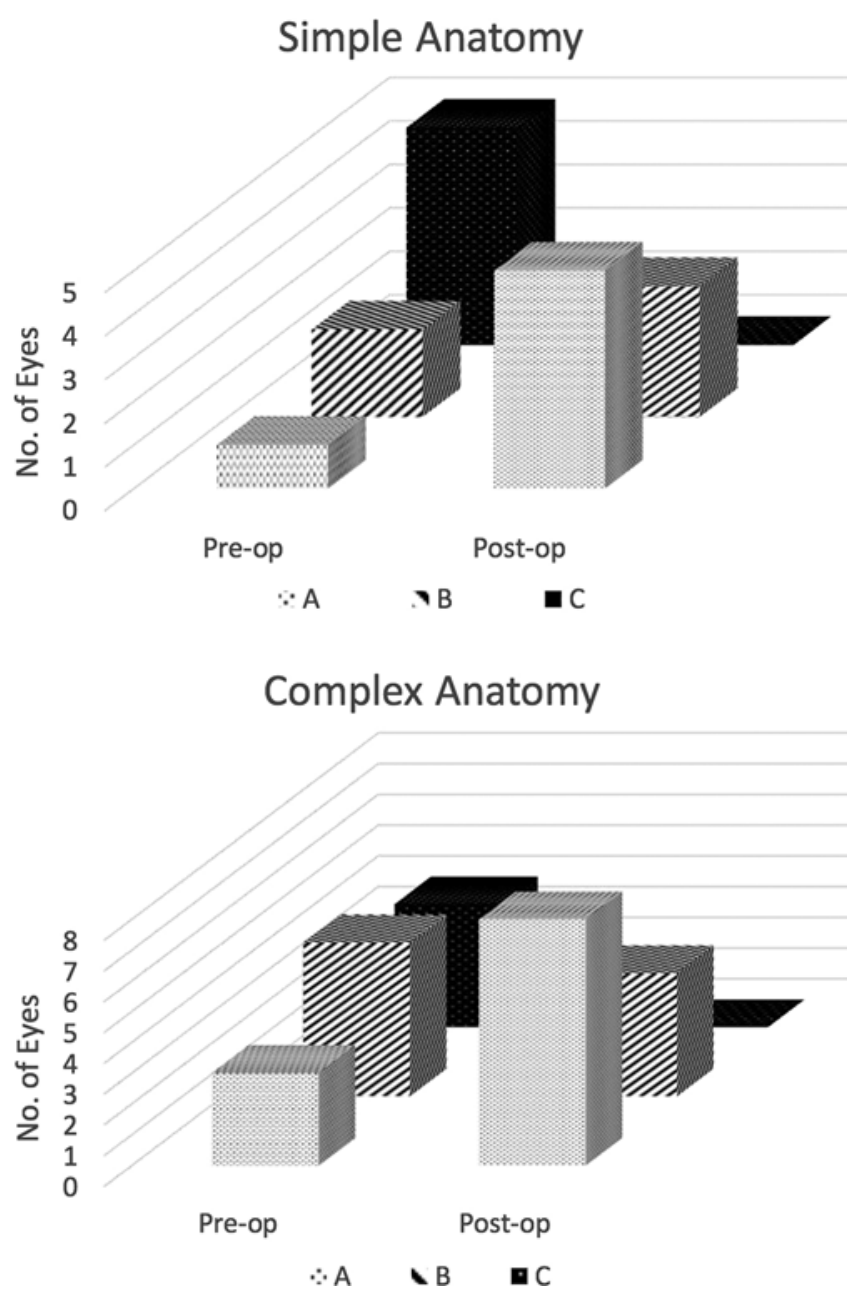

FIG. 2. Bar graph of visual changes using UVFS grades $A-C$ in patients with perisellar meningiomas for those with simple anatomy ( $n=8$ eyes) and complex anatomy ( $n=12$ eyes).

previously mentioned methods for comparison. VL was calculated as described previously by Rosenstein and Symon. ${ }^{33}$ Each eye was assessed individually with respect to the state of visual fields and visual acuity. Isolated monocular blindness with no loss of vision in the opposite eye was given a score of $52.5 \%$ to take into the account the loss of stereoscopic vision. A patient with binocular blindness had a score of $100 \%$, while one with no loss of vision had a score of 0 . A VIQ was derived from a ratio of pre- and postoperative VL (Table 2). Patients with visual improvement have positive VIQ values between 0 and 1, the latter representing $100 \%$ improvement. Patients with visual deterioration have negative values. We also used the German Ophthalmological Society scale to calculate the VIS (Fig. 1). Across the cases, we noted a correlation between the VIQ and the VIS (Tables 4 and 5). Visual changes were accurately captured among the 3 scales compared.

\section{Discussion}

\section{Rationale}

Uniform reporting of visual outcomes in clinical trials would be advantageous for accurate appraisal of medical 
TABLE 4. Comparison of visual function scales in 10 sample patients with perisellar meningiomas that had simple anatomy

\begin{tabular}{|c|c|c|c|c|c|c|c|c|c|c|}
\hline \multirow[b]{3}{*}{ Case No. } & \multicolumn{4}{|c|}{ UVFS Grade } & \multirow{2}{*}{\multicolumn{3}{|c|}{$\mathrm{VL}(\mathrm{VIQ})^{*}$}} & \multirow{2}{*}{\multicolumn{3}{|c|}{$\begin{array}{c}\text { German Ophthalmological } \\
\text { Society Score (VIS) } \dagger\end{array}$}} \\
\hline & \multicolumn{2}{|c|}{ Preop } & \multicolumn{2}{|c|}{ Postop } & & & & & & \\
\hline & $O D$ & OS & $O D$ & OS & Preop & Postop & VIQ & Preop & Postop & Change \\
\hline 1 & C & A & $B$ & A & 67.5 & 22.5 & 0.67 & 56 & 13 & +43 \\
\hline 2 & A & A & A & A & 22.5 & 22.5 & 0.00 & 22 & 22 & 0 \\
\hline 3 & $A$ & C & $A$ & $B$ & 35 & 20 & 0.43 & 34 & 11 & +23 \\
\hline 4 & $B$ & A & $A$ & A & 15 & 0 & 1.00 & 11 & 0 & +11 \\
\hline 5 & $A$ & C & A & A & 25 & 5 & 0.8 & 32 & 4 & +28 \\
\hline 6 & A & A & A & A & 10 & 0 & 1.00 & 6 & 0 & +6 \\
\hline 7 & A & B & A & A & 10 & 5 & 0.50 & 9 & 4 & +5 \\
\hline 8 & C & $A$ & $A$ & $A$ & 15 & 0 & 1.00 & 20 & 0 & +20 \\
\hline 9 & $A$ & C & $A$ & $A$ & 47.5 & 5 & 0.89 & 57 & 8 & +49 \\
\hline 10 & $A$ & $A$ & B & B & 0 & 15 & NA & 4 & 18 & -14 \\
\hline
\end{tabular}

$\mathrm{OD}=$ oculus dexter; $\mathrm{OS}=$ oculus sinister; $\mathrm{NA}=$ not applicable.

* From Rosenstein and Symon. ${ }^{33}$

$\dagger$ From Fahlbusch and Schott, ${ }^{14}$ and Jaeger and Thomann. ${ }^{20}$

literature relating to vision. Without standard guidelines for reporting visual outcomes, data comparison between studies is compromised, and pooling of data for metaanalysis is challenging, if not impossible. No specific uniform classification system currently exists, as it is extremely challenging to group a heterogeneous population of patients into fixed categories, because data resolution will be diminished. This loss of resolution must be balanced against applicability for daily clinical use. Additionally, the functional impact of the disease should be quantified in a clinically and functionally useful manner, as this would provide meaningful information for a clinician when assessing impact of an intervention. With these ideas in mind, a new visual outcome scale for clinical use has been created. This comprehensive scale is intended for daily use by surgeons for assessing VL in the forms of visual acuity and visual fields.

The combination of visual acuity and fields for a net score to describe vision is not a novel concept and has been previously used to assess outcomes following surgery by Fahlbusch and Schott ${ }^{14}$ and Rosenstein and Symon. ${ }^{33}$ These assessments have been used to adequately quantify degrees of visual change in the postoperative period, but much like the VFQ-25 and the AVDS, they are more suited for use outside of the clinical setting. Similar to assessing hearing outcomes, a visual scale is needed that has ease of use, similar to the American Academy of Otolaryngology-Head and Neck Surgery and GardnerRobertson scales. ${ }^{15,29}$ When comparing the 2 visual function scales currently available to the UVFS, we demonstrate that there is sufficient sensitivity of the UVFS to detect direct clinically meaningful and easily understandable changes to vision, either improvement or worsening (Tables 4 and 5).

The abstract definitions and point systems of the German Ophthalmological Society and Fahlbusch's combined visual scales to define degrees of visual impairment have been neither validated nor tested for interobserver reli-

TABLE 5. Comparison of visual function scales in 10 sample patients with perisellar meningiomas with complex anatomy

\begin{tabular}{|c|c|c|c|c|c|c|c|c|c|c|}
\hline \multirow{3}{*}{$\begin{array}{l}\text { Case } \\
\text { No. }\end{array}$} & \multicolumn{4}{|c|}{ UVFS Grade } & \multirow{2}{*}{\multicolumn{3}{|c|}{$\mathrm{VL}(\mathrm{VIQ})^{*}$}} & \multirow{2}{*}{\multicolumn{3}{|c|}{$\begin{array}{l}\text { German Ophthalmological } \\
\text { Society Score (VIS)† }\end{array}$}} \\
\hline & \multicolumn{2}{|c|}{ Preop } & \multicolumn{2}{|c|}{ Postop } & & & & & & \\
\hline & OD & OS & $O D$ & OS & Preop & Postop & $V I Q$ & Preop & Postop & Change \\
\hline 1 & A & C & B & C & 40 & 65 & -0.63 & 47 & 48 & -1 \\
\hline 2 & C & C & A & A & 75 & 10 & 0.87 & 90 & 10 & +80 \\
\hline 3 & A & A & B & A & 20 & 15 & 0.25 & 19 & 20 & -1 \\
\hline 4 & C & A & C & B & 25 & 70 & -1.80 & 37 & 57 & -20 \\
\hline 5 & B & A & C & B & 22.5 & 75 & -2.33 & 20 & 52 & -32 \\
\hline 6 & B & B & A & A & 15 & 5 & 0.67 & 22 & 8 & +14 \\
\hline 7 & A & A & A & B & 5 & 22.5 & -3.50 & 6 & 27 & -21 \\
\hline 8 & C & C & A & B & 47.5 & 30 & 0.37 & 81 & 32 & +49 \\
\hline 9 & A & B & A & A & 22.5 & 12.5 & 0.44 & 27 & 6 & +21 \\
\hline 10 & A & B & A & A & 10 & 10 & 0.00 & 5 & 10 & -5 \\
\hline
\end{tabular}

${ }^{*}$ From Rosenstein and Symon. ${ }^{33}$

$\dagger$ From Fahlbusch and Schott, ${ }^{14}$ and Jaeger and Thomann. ${ }^{20}$ 
ability. The arbitrary boundaries that separate different scoring groups for visual impairment are difficult to correlate clinically and to understand. While it appears they are sufficiently granular to describe the diversity of visual outcomes, their clinical application is cumbersome. The values obtained from calculations for VL and VIS may be useful to compare patient groups with each other from a research perspective. In isolation, however, they do not offer significant insight into the overall degree of visual change, much like the descriptors of "improved," "worsened," and "unchanged." For example, a patient with a quadrantanopsia would have the same VL score as someone with loss of 6/9 according to the Rosenstein scale (both score 1; Table 2). The functional difference between these 2 outcomes, however, can be dramatic, such as when considering driving with monocular vision.

With our goal to design the UVFS to be straightforward and intuitive, we hope to be able to accurately attribute degrees of impact for visual outcomes following surgical intervention for lesions affecting the optic apparatus. We chose legal blindness and fitness to drive as our cutoffs, such that there is significant functional implication with each grade. We believe shifts between grades would have direct consequences on patient quality of life. For example, while at first glance a clinician may not directly appreciate a visual acuity improvement from $20 / 70$ to $20 / 50$, this is a change from grade B to grade A on the UVFS, suggesting a possibility that the patient may now qualify for reassessment of clearance to drive a private vehicle. Conversely, a patient may not undergo significant changes to his or her visual acuity, but perhaps due to visual field changes becomes ineligible to operate a private vehicle. This would likely represent a significant decrease in his or her quality of life and would be captured by an assessment with the UVFS. Additionally, we believed that recording minor changes to either visual acuity or visual fields would be overwhelming for a clinical assessment scale; a slight improvement of a few degrees to a patient with a homonymous hemianopsia field deficit would not be of clinical significance. When comparing the 2 currently available visual function scales to the UVFS, we demonstrated that there is sufficient sensitivity of the UVFS to detect direct changes to visual improvement or worsening (Tables 4 and 5), whereas the clinical impact of the VIQ or VIS as per the German Ophthalmological Society is difficult to interpret.

The problems we encountered with the German Ophthalmological Society scale used in the paper by Fahlbusch and Schott are similarly applicable to Rosenstein's scale, whereby arbitrary values for visual acuity and fields are combined to give an overall score for visual impairment. After calculating the VISs, Rosenstein implements the VIQ to "evaluate quantitatively the effects of surgery on vision." "33 However, the VIQ is difficult to interpret. For example, patient 1 with a complex anatomy lesion experienced vision deterioration in the postoperative period from grade A to grade B in the right eye (Table 5). This is a significant clinical implication as the patient is legally blind in the left eye, which remained unchanged. The VIS, however, was calculated as -1 , which does not appear to be a significant degree of change. In contrast, patient 7 with a complex tumor also had visual worsening in the left eye, from grade A to grade B, but the VIS was calculated to be -21 (Table 5). Given the right eye remained unchanged with good function, the clinical impact may be minor as overall the patient would not be prohibited from driving. In addition, the importance of several of the deficits is underscored as a result of the arbitrary nature of the scale. For example, there is not much distinction for clinicians if deficits occur only in 1 eye, as the VIS is a reflection of visual function in both eyes. This information is not translated when examining VISs in isolation without clinical history. The varying degrees in visual field restriction are graded as less disabling than changes of visual acuity. Ultimately, like the German Ophthalmological Society scale, the Rosenstein scale presents visual impairment values that have poor clinical correlation, and are cumbersome to use.

\section{Scale Limitations}

Our visual assessment scale is based on the strict definitions of legal blindness and fitness-to-drive requirements in Canada. Although these are arbitrary cutoffs for the grades of visual function impairment in Canada, they are reasonably generalizable. The definition of legal blindness in terms of visual acuity and visual fields appears to be quite uniform across the US and Europe.,.$^{2,410}$ The cutoffs for requirements to drive a private vehicle have some minor variability (Table 6). European countries generally follow the European Union driver's license requirements (20/40 visual acuity, binocular visual fields of $120^{\circ}$ ) with some minor adjustments (Table 6), ${ }^{4,10,12}$ The visual acuity requirements for holding a driver's license in the US are state dependent but are similar to those in Europe (Table 6). There is more variability in visual field requirements among different jurisdictions, which can be demonstrated in 3 arbitrarily chosen states (California, Iowa, and New York; Table 6).,1,30 Evidence suggests that those drivers with impaired visual acuity are more likely to make errors identifying highway signs at a distance. ${ }^{19}$ This is not surprising given that type size for signs is selected such that the sign can be effectively read at appropriate braking distances by people who have at least $20 / 40$ acuity or better. ${ }^{34}$ We believed our conservative cutoffs for visual acuity and visual fields would not only be applicable for clinical use at our facilities, but also, given the similarity to international regulations, allow for extension of generalizability to other institutions and countries. However, by basing our scale around the definitions of legal blindness and fitness to drive, the UVFS would fail to capture cases in which clinically significant or patient-centered visual quality of life improves or deteriorates but does not qualify for a categorical shift within the UVFS.

Use of the scale also necessitates the assessment of each eye separately, as combining the visual acuity and fields from both eyes certainly does not have the accuracy to evaluate for visual changes affecting 1 eye only. The utility of a scale strongly relies on its ease of use, especially when gauged for suitability within both clinical and research environments. We believed we needed to ensure that the UVFS remained simple while still maintaining a reasonable resolution, which is why we chose to assign a grade to each eye without combining them. Despite this, it should be noted that definitions for legal blindness and fitness-todrive evaluations are performed with both eyes combined. 
TABLE 6. International driver's license requirements

\begin{tabular}{|c|c|c|}
\hline Country & Visual Acuity* & Visual Field $†$ \\
\hline Canada & $20 / 50$ & At least $120^{\circ}$ along the horizontal, $15^{\circ}$ above \& below \\
\hline US & $20 / 40$ & Some states none, most states $110^{\circ}-140^{\circ}$ \\
\hline California & $20 / 40$ & No visual field requirement \\
\hline New York & $20 / 40$ in at least 1 eye & If visual acuity is btwn $20 / 40 \& 20 / 70$, at least $140^{\circ}$ \\
\hline lowa & $20 / 40$ in at least 1 eye & $140^{\circ}$ or better \\
\hline United Kingdom & $20 / 40$ & At least $120^{\circ}$ \\
\hline Germany & At least $20 / 40$ in better eye, $20 / 100$ in worse eye & At least $120^{\circ}\left(\right.$ perfect w/in $\left.30^{\circ}\right)$ \\
\hline France & $20 / 40$ & At least $60^{\circ}$ along the horizontal, $30^{\circ}$ above $\&$ below \\
\hline Spain & $20 / 40$ & Visual field must be normal (at least $120^{\circ}$ ) \\
\hline Italy & $20 / 20$ & Visual field must be normal (at least $120^{\circ}$ ) \\
\hline Australia & $20 / 40$ & At least $110^{\circ}$ along the horizontal, $10^{\circ}$ above $\&$ below \\
\hline
\end{tabular}

\section{Study Limitations}

The UVFS is a scale that was established using a combination of visual acuity and visual field results to gauge visual outcomes. Its use was demonstrated in a case series from a single-center retrospective chart review. The strength of our scale is limited by the chance of the findings not being generalizable. While the cutoff points for visual acuity and visual fields that have been selected are applicable to laws and regulations in Canada where the authors practice, and may not be easily applicable across the world, we believe that they are reasonable cutoff points for creating categories. When comparing the requirements in various jurisdictions, they are relatively similar (Table 6), and in practical use, most jurisdictions allow use of driver's licenses from other jurisdictions when traveling, for example. The scale also may not be sensitive enough to identify patients experiencing minor changes in visual acuity or fields, but with significant impact on the quality of life, such as loss of stereoscopic vision. Additionally, the use of the UVFS was not explored in patients with lesions affecting other parts of the optic apparatus outside of the optic nerve, tracts, and chiasm. Finally, in Tables 4 and 5, the selection of 10 patients from the case series as an illustrative cohort for use in comparison with previous scales was also determined on a subjective and convenience basis.

\section{Future Directions}

Given that our scale is based on the functional impact of visual outcomes, validation with quality of life is necessary. Correlation of the scale grades with the AVDS and VFQ-25 would strengthen its applicability in the clinical setting. Additionally, confirmation of interobserver reliability would strengthen the applicability of the scale.

\section{Conclusions}

Currently no universal standard exists for reporting visual results following surgical intervention. We present a scale that offers a grading system based around functional impact that is practical for daily clinical assessments. The UVFS will additionally allow for more efficient comparison and pooling of data for meta-analysis. The UVFS would be strengthened once correlated with direct qualityof-life assessments that would improve its application in a neurosurgical environment.

\section{References}

1. American Association of Motor Vehicle Administrators: Driver Fitness Medical Guidelines. (http://ntl.bts.gov/ lib/31000/31100/31148/6061_MedicalReviewGuide_10-1_ v2a.pdf) [Accessed September 12, 2017]

2. American Foundation for the Blind: Key definitions of statistical terms. (http://www.afb.org/info/blindnessstatistics/key-definitions-of-statistical-terms/25) [Accessed September 12, 2017]

3. Benjamini Y, Hochberg Y: Controlling the false discovery rate: a practical and powerful approach to multiple testing. J R Stat Soc B 57:289-300, 1995

4. Bron AM, Viswanathan AC, Thelen U, de Natale R, Ferreras A, Gundgaard J, et al: International vision requirements for driver licensing and disability pensions: using a milestone approach in characterization of progressive eye disease. Clin Ophthalmol 4:1361-1369, 2010

5. Canadian Association of Optometrists: Position Statement on Low Vision and Blindness. (https://opto.ca/sites/default/ files/cao_position_statement_-_low_vision_and_blindness. pdf) [Accessed September 12, 2017]

6. Canadian Council of Motor Transport Administrators: Medical Standards for Drivers with BC Specific Guidelines. (http://www2.gov.bc.ca/assets/gov/drivingand-transportation/driving/publications/2016-ccmta-guide. pdf) [Accessed September 12, 2017]

7. Canadian Medical Association: CMA Driver's Guide: Determining Medical Fitness to Operate Motor Vehicles. (https://joule.cma.ca/en/evidence/CMA-drivers-guide.html) [Accessed September 12, 2017]

8. Chokyu I, Goto T, Ishibashi K, Nagata T, Ohata K: Bilateral subfrontal approach for tuberculum sellae meningiomas in long-term postoperative visual outcome. J Neurosurg 115:802-810, 2011

9. Ciric I, Rosenblatt S: Suprasellar meningiomas. Neurosurgery 49:1372-1377, 2001

10. Driver and Vehicle Licensing Agency: Driving eyesight rules. (https://www.gov.uk/driving-eyesight-rules) [Accessed September 12, 2017]

11. Ehlers N, Malmros R: The suprasellar meningioma. A review of the literature and presentation of a series of 31 cases. Acta Ophthalmol Suppl 1973:1-74, 1973 
12. European Commission: Council directive 91/439/EEC of 1991 Jul 29 on driving licenses. (http://eur-lex.europa.eu/ legal-content/EN/TXT/?uri=CELEX:31991L0439) [Accessed September 12, 2017]

13. Ezzat S, Asa SL, Couldwell WT, Barr CE, Dodge WE, Vance ML, et al: The prevalence of pituitary adenomas: a systematic review. Cancer 101:613-619, 2004

14. Fahlbusch R, Schott W: Pterional surgery of meningiomas of the tuberculum sellae and planum sphenoidale: surgical results with special consideration of ophthalmological and endocrinological outcomes. J Neurosurg 96:235-243, 2002

15. Gardner G, Robertson JH: Hearing preservation in unilateral acoustic neuroma surgery. Ann Otol Rhinol Laryngol 97:55-66, 1988

16. Glickman ME, Rao SR, Schultz MR: False discovery rate control is a recommended alternative to Bonferroni-type adjustments in health studies. J Clin Epidemiol 67:850-857, 2014

17. Goel A, Muzumdar D, Desai KI: Tuberculum sellae meningioma: a report on management on the basis of a surgical experience with 70 patients. Neurosurgery 51:1358-1364, 2002

18. Gregorius FK, Hepler RS, Stern WE: Loss and recovery of vision with suprasellar meningiomas. J Neurosurg 42:69-75, 1975

19. Higgins KE, Wood J, Tait A: Vision and driving: selective effect of optical blur on different driving tasks. Hum Factors 40:224-232, 1998

20. Jaeger W, Thomann H: Deutsche Ophthalmologische Gesellschaft. Empfehlungen zur Beurteilung der Minderung der Erwebsfähigkeit durch Schäden des Sehvermögens. Klin Mbl Augenheilk 180:242-245, 1982

21. Jallo GI, Benjamin V: Tuberculum sellae meningiomas: microsurgical anatomy and surgical technique. Neurosurgery 51:1432-1440, 2002

22. Kadis GN, Mount LA, Ganti SR: The importance of early diagnosis and treatment of the meningiomas of the planum sphenoidale and tuberculum sellae: a retrospective study of 105 cases. Surg Neurol 12:367-371, 1979

23. Koutourousiou M, Fernandez-Miranda JC, Stefko ST, Wang EW, Snyderman CH, Gardner PA: Endoscopic endonasal surgery for suprasellar meningiomas: experience with 75 patients. J Neurosurg 120:1326-1339, 2014

24. Mahmoud M, Nader R, Al-Mefty O: Optic canal involvement in tuberculum sellae meningiomas: influence on approach, recurrence, and visual recovery. Neurosurgery 67 (3 Suppl Operative):ons108-ons119, 2010

25. Makarenko S, Carreras EM, Akagami R: Craniotomy for perisellar meningiomas: comparison of simple (appropriate for endoscopic approach) versus complex anatomy and surgical outcomes. J Neurosurg 126:1191-1200, 2017

26. Mangione CM, Lee PP, Gutierrez PR, Spritzer K, Berry S, Hays RD: Development of the 25-item National Eye Institute Visual Function Questionnaire. Arch Ophthalmol 119:1050-1058, 2001

27. Mangione CM, Phillips RS, Seddon JM, Lawrence MG, Cook EF, Dailey R, et al: Development of the 'Activities of Daily Vision Scale'. A measure of visual functional status. Med Care 30:1111-1126, 1992

28. Ministry of Public Safety and Solicitor General Office of the Superintendent of Motor Vehicles: 2010 BC Guide in Determining Fitness to Drive. (http://www2.gov. bc.ca/assets/gov/driving-and-transportation/driving/ publications/2010-guide-in-determining-fitness-to-drive.pdf) [Accessed September 12, 2017]
29. Monsell EM: New and revised reporting guidelines from the Committee on Hearing and Equilibrium. Otolaryngol Head Neck Surg 113:176-178, 1995

30. New York State Department of Motor Vehicles: Vision requirements \& restrictions. (https://dmv.ny.gov/ driver-license/vision-requirements-restrictions) [Accessed September 12, 2017]

31. Ray WA, O'Day DM: Statistical analysis of multi-eye data in ophthalmic research. Invest Ophthalmol Vis Sci 26:11861188,1985

32. Reisch R, Perneczky A: Ten-year experience with the supraorbital subfrontal approach through an eyebrow skin incision. Neurosurgery 57 (4 Suppl):242-255, 2005

33. Rosenstein J, Symon L: Surgical management of suprasellar meningioma. Part 2: Prognosis for visual function following craniotomy. J Neurosurg 61:642-648, 1984

34. Schieber F: Highway research to enhance safety and mobility of older road users, in Transportation in an Aging Society: A Decade of Experience. Washington, DC: Transportation Research Board, 2004, pp 125-154

35. Shields JA, Shields CL, Scartozzi R: Survey of 1264 patients with orbital tumors and simulating lesions: The 2002 Montgomery Lecture, part 1. Ophthalmology 111:997-1008, 2004

36. Stefko ST, Snyderman C, Fernandez-Miranda J, Tyler-Kabara E, Wang E, Bodily L, et al: Visual outcomes after endoscopic endonasal approach for craniopharyngioma: the Pittsburgh experience. J Neurol Surg B Skull Base 77:326-332, 2016

37. Wilson DAD, Duong H, Teo C, Kelly DF: The supraorbital endoscopic approach for tumors. World Neurosurg 82 (6 Suppl):S72-S80, 2014

\section{Disclosures}

The authors report no conflict of interest concerning the materials or methods used in this study or the findings specified in this paper.

\section{Author Contributions}

Conception and design: Makarenko, Gooderham, Akagami. Acquisition of data: Makarenko, Ye. Analysis and interpretation of data: Makarenko, Ye, Akagami. Drafting the article: Makarenko, Ye, Akagami. Critically revising the article: all authors. Reviewed submitted version of manuscript: all authors. Approved the final version of the manuscript on behalf of all authors: Makarenko. Statistical analysis: Makarenko. Administrative/technical/ material support: Makarenko. Study supervision: Gooderham, Akagami.

\section{Supplemental Information}

\section{Previous Presentations}

Portions of this work were presented in abstract form and as proceedings at the 27th Annual Meeting of the International Conference on Skull Base Surgery, North American Skull Base Society, in New Orleans, Louisiana, March 3, 2017.

\section{Correspondence}

Serge Makarenko: Vancouver General Hospital, Vancouver, BC, Canada.smakarenko@gmail.com. 